\title{
Linx
}

Revue des linguistes de l'université Paris X Nanterre

$42 \mid 2000$

Approches sociolinguistiques du plan phonique

\section{Une forme linguistique de dialogue spécifique : le cas de l'interaction homme-machine sur terminal}

\section{Thierry Wabl}

\section{OpenEdition}

\section{Journals}

Édition électronique

URL : http://journals.openedition.org/linx/912

DOI : $10.4000 / \operatorname{linx} .912$

ISSN : 2118-9692

Éditeur

Presses universitaires de Paris Nanterre

\section{Édition imprimée}

Date de publication : 1 juin 2000

Pagination : 159-174

ISSN : 0246-8743

\section{Référence électronique}

Thierry Wabl, « Une forme linguistique de dialogue spécifique : le cas de l'interaction homme-machine sur terminal », Linx [En ligne], 42 | 2000, mis en ligne le 05 juillet 2012, consulté le 20 avril 2019. URL: http://journals.openedition.org/linx/912 ; DOI : 10.4000/linx.912

Ce document a été généré automatiquement le 20 avril 2019.

Département de Sciences du langage, Université Paris Ouest 


\title{
Une forme linguistique de dialogue spécifique : le cas de l'interaction homme-machine sur terminal
}

\author{
Thierry Wabl
}

\section{Introduction}

1 Cet article s'inscrit dans le cadre des recherches sur l'interaction entre l'homme et la machine, par le biais du langage. Brièvement, il convient de préciser que notre travail répond à une demande industrielle puisqu'il s'agissait initialement d'améliorer une interface de dialogue afin d'aider les utilisateurs à retrouver l'information contenue dans une base de données bibliographiques. Nous n'abordons pas ici les caractéristiques techniques de l'interface ni celles de l'expérimentation. Cependant, dès à présent, il faut noter que certains phénomènes observés peuvent s'expliquer tout d'abord par le fait que les utilisateurs ne sont pas habitués ou familiarisés à ce type d'interaction et donc, peutêtre, n'ont-ils pas encore modifié leurs pratiques d'écrit comme certains ont pu le constater dans d'autres corpus à propos d'échanges similaires (D. LUZZATI 1989, 1991, M.A. MOREL 1988, 1989).

2 Les remarques et analyses qui vont suivre reposent sur l'étude d'un corpus de dialogues, recueilli au cours d'une simulation expérimentale, entre un utilisateur et une machine lors de l'interrogation d'une base de données bibliographiques. Il est constitué de 21 consultations de longueurs variables. L'humain interroge une interface qui doit l'aider à retrouver l'information qu'il désire. Notre problématique est donc de considérer le dialogue homme-machine (désormais $\mathrm{DHM}^{1}{ }^{1}$ comme un terrain possible de la sociolinguistique dans lequel les mécanismes de l'interaction entre utilisateurs et concepteurs relèvent d'une co-production du sens socialisée, situation commune à toute production de discours, nous semble-t-il. L'analyse du corpus consiste en l'étude des 
phénomènes linguistiques du DHM, concernant ici plus spécifiquement les manifestations de l'écrit et de l'oral.

Aussi il convient de se demander comment l'on peut définir les interactions entre homme et machine que nous avons recueillies? Relèvent-elles davantage de l'oral ou de l'écrit? Est-ce un dialogue ou une communication écrite?

Il faut remarquer tout d'abord que cette interaction est une structure d'échange dans laquelle les participants ont, au moins théoriquement, la possibilité de devenir énonciateurs. D'après la typologie élaborée par R. VION (1992 : 119-141), elle entre dans la catégorie de la consultation, qui est une relation d'aide, dans laquelle il y a adaptation de l'utilisateur à la machine.La consultation, par la nature de ses finalités, est orientée vers le domaine de l'action et de la connaissance. Bien que ce soit une interaction hommemachine (désormais IHM), elle possède des traits (en fait dus essentiellement à l'usager) qui la rapprochent du dialogue.

5 Les travaux antérieurs, notamment ceux de J. ANIS (1993, 1994, 1998), ont montré la place de l'écrit ainsi que les caractéristiques générales de ce type de communication. L'intérêt pour nous est d'en définir les particularités sur un corpus en situation. Aussi s'agit-il de revenir sur cet écrit particulier afin de voir ce qui le caractérise d'un point de vue graphématique.

\section{La structure de communication : un modèle de dialogue sur terminal}

\subsection{Les constituants du dialogue sur terminal}

Ce mode de communication implique des éléments constitutifs de cet échange. Il s'agit :

- d'un outil d'écriture : le clavier de l'ordinateur

- d'un support de lecture/écriture : l'écran de l'ordinateur

- du message qui est émis et lu par un seul locuteur

- d'un léger différé vécu comme « une quasi-immédiateté avec le même sentiment d'urgence que dans un échange direct » (J. ANIS 1994 : 85).

7 Notons aussi que les conditions de recueil du corpus peuvent elles aussi avoir eu une importance capitale. Les utilisateurs ont très certainement fait quelques efforts dans leur pratique du fait de notre présence. On n'écrit pas de la même façon dans ce contexte que seul devant une machine lorsque les écrits sont totalement anonymes et disparaissent. Enfin la gratuité de la connexion ne les obligeait pas à faire court et/ou concis.

\subsection{Les limites des possibilités d'expression imposées par l'interface}

8 Le décalage de sens constaté dans les échanges de l'utilisateur avec ceux de la machine résulte souvent des possibilités d'expression que le système impose. Le principe de concision dans les échanges de l'utilisateur procède du fait que l'humain oralise son écrit et que la langue orale possède ses propres types d'économie linguistique puisque sa syntaxe est différente de celle de l'écrit. Les échanges semblent souvent réduits à leur plus simple expression comme l'illustreront ces deux exemples ${ }^{2}$ : 


\begin{tabular}{|l|l|}
\hline 10M11 & AUCUNE FICHE, JE SUIS VRAIMENT NAVRÉ \\
\hline $10 H 11$ & PAS GRAVE SALUT \\
\hline $17 M 8$ & LAISSEZ VOUS GUIDER POUR UN NOUVEAU THÈME... \\
\hline $17 H 8$ & UN TRUC SUR L'THÉÂTRE \\
\hline $17 M 9$ & UN OUVRAGE SUR « DISCOURS LITTÉRAIRE » C'EST CELA? \\
\hline $17 H 9$ & OUI ÇA PEUT L'FAIRE JE CROIS \\
\hline
\end{tabular}

9 Cependant, la machine est le plus souvent l'acteur de ces réductions ou économies linguistiques, car la mise en mémoire de termes qui sont retournés à l'utilisateur sous forme de questions, peut provoquer ces processus d'économie. Par ce type de réponse de la machine, l'utilisateur comprend sans doute que la recherche s'effectue sur quelques « termes clefs » et non sur l'ensemble de la phrase. Exemple :

\begin{tabular}{|l|l|}
\hline $18 M 8$ & UN OUVRAGE SUR «DISCOURS JURIDIQUE » C'EST CELA? \\
\hline \hline $18 H 8$ & C'EST MOI QUI VOUS SUIT PLUS MAINTENANT \\
\hline $18 \mathrm{M} 9$ & « MAINTENANT»? \\
\hline $18 \mathrm{H} 9$ & OU JAMAIS \\
\hline
\end{tabular}

10 Les quatre Maximes conversationnelles proposées par H. P. GRICE (1975), à savoir « la Quantité, la Qualité, la Pertinence et la Manière » prennent alors tout leur sens et sont à intégrer dans le principe général de la coopération, principe selon lequel l'énonciateur est supposé ne pas bafouer les maximes. La théorie de GRICE repose entièrement sur l'hypothèse selon laquelle le comportement interprétatif est dirigé par les intentions du locuteur. Pour réussir sa communication le locuteur se doit de respecter le principe de coopération et ces maximes de conversation érigés en règles implicites de toute communication verbale "réussie ». Afin d'être compris par autrui, le sens d'un message est lié de la sorte à la communication des intentions du locuteur. Les références au contexte ou à la situation d'énonciation sont centrales. Les principes généraux établis, il reste à étudier comment ceux-ci se traduisent linguistiquement dans une communication homme-machine.

\section{Les principales caractéristiques de cet échange}

11 Il est important de déterminer ce qui, dans cet échange, est conditionné par la communication écrite de ce qui relève du dialogue. C'est un fait: cet échange est effectivement écrit. Il n'y a par conséquent ni intonation, ni paraverbal, ni de phénomène de simultanéité comme dans tout oral! De plus, cet échange intègre les caractéristiques propres de l'écrit comme nous allons le voir maintenant. 


\subsection{Les marques de l'écrit}

\begin{tabular}{|l|l|}
\hline 13M1 & BONSOIR, JE VAIS ESSAYER DE VOUS GUIDER DANS VOTRE RECHERCHE, QUE DÉSIREZ-VOUS ? \\
\hline $13 H 1$ & J'AIMERAIS CONNAÎTRE L'AUTEUR D'UN TRAITÉ DE DROIT CIVIL \\
\hline $13 M 2$ & UN OUVRAGE SUR « DISCOURS JURIDIQUE » C'EST CELA? \\
\hline $13 H 2$ & OUI À PEU PRÈS \\
\hline $13 M 3$ & J'AI PLUS DE CINQ FICHES SUR LA QUESTION, LES VOULEZ-VOUS TOUTES ? \\
\hline $13 H 3$ & NON L'AUTEUR EST NÉ DANS LES ANNÉES TENTE \\
\hline $13 M 4$ & APPORTEZ-MOI QUELQUES PRÉCISIONS SUR CE TERME « DISCOURS JURIDIQUE », JE VOUS PRIE. \\
\hline 13H4 & JE SOUHAITERAIS CONNAÎTRE L'OPINION DE CET AUTEUR SUR UN TERME JURIDIQUE \\
\hline
\end{tabular}
vérification de l'orthographe de termes utilisés par l'utilisateur. Il s'agit bien d'écrit. Exemple :

\begin{tabular}{|l|l|}
\hline $14 M 11$ & ÉNONCEZ UN THÈME DE RECHERCHE... \\
\hline $14 H 11$ & SUR BENVENSITE \\
\hline \hline $14 M 12$ & VOUS VOULEZ DIRE « BENVENISTE »? \\
\hline
\end{tabular}
donc là un travail de brouillon avec effacement, sans trace.

16 Cet écrit s'exerce en direct, avec un temps de transmission très court. Ce qui entraîne de nombreux faits qui sont eux aussi spécifiques à cet échange :

17 les abréviations il n'existe dans le corpus que peu de réduction de mots à leur transcription consonantique comme il en existe habituellement dans les interactions minitéliques entre usagers (exemple : K pour que). En revanche nous avons noté que les usagers utilisent certaines abréviations usuelles à l'oral et non spécifiques de ce type d'écrits :

\begin{tabular}{|l|l|}
\hline 7 7H9 & ET BIEN SUR LES MÉTHODOS SCIENTIFIQUES \\
\hline \hline $19 H 9$ & IMPEC... \\
\hline
\end{tabular}


Certains mots sont réduits à leur plus simple expression qui sont les «squelettes consonantiques »: SLT pour salut, BJ pour bonjour :

10H1 SLT, JE VOUDRAIS DES TRAVAUX SUR L'HYPERTEXTE

19 L'alternance des tours de parole : dans notre corpus, elle est mécanique et il ne peut en être autrement. Seule l'attente prolongée d'une réponse de l'utilisateur peut signifier soit un refus de communiquer, soit le désir du prolongement de son tour de parole. Ainsi pour qu'il y ait présence d'un silence, il faut que l'utilisateur produise volontairement et explicitement un «blanc » (en appuyant sur la touche envoi comme le montre l'exemple 1; ce blanc est transcrit dans notre corpus par un «/»). C'est donc là une particularité de ce dialogue qui marque une différence importante avec une interaction naturelle. Une autre grande différence réside dans le fait que le silence de l'utilisateur, non marqué formellement et intentionnellement, n'est pas interprété par la machine. Le silence dans ce cas n'existe pas. Nous sommes soucieux ici de proposer ultérieurement la possibilité pour la machine de récupérer le tour de parole lorsque l'utilisateur met un certain temps (déterminé à l'avance) pour répondre ; ce n'est pas le cas ici.

- L'absence de pause: il nous faut ici préciser le fonctionnement de l'interface concernant cette question. Dans cet échange, il n'y a pas de pause c'est-à-dire de suspension momentanée des énoncés. Quand l'humain a fini son énoncé, il le valide à l'aide de la touche envoi, indiquant par là même explicitement qu'il a fini. S'il ne le valide pas, le système est «bloqué », la parole reste à l'usager. Enfin, lorsque l'humain demande une pause de façon implicite (cf exemple 1) ou explicite (exemple 2), celle-ci lui est refusée automatiquement par la machine qui répond et relance l'échange car elle n'intègre pas la demande de pause. L'interface fonctionne comme une personne qui refuse les silences que l'autre demande. C'est pourquoi le seul moyen de faire une pause est de faire un silence en validant son tour de parole sans rien inscrire, mais hélas la machine le repère.

Exemple 1:

\begin{tabular}{|l|l|}
\hline $9 \mathrm{M} 4$ & une autre recherche peut-être? \\
\hline $9 \mathrm{H} 4$ & / \\
\hline \hline $9 \mathrm{M} 5$ & écrivez-moi quelque chose sinon votre recherche va tourner court ! \\
\hline $9 \mathrm{H} 5$ & pardon je réfléchissais \\
\hline
\end{tabular}

Exemple 2:

\begin{tabular}{|l|l|}
\hline $20 \mathrm{H} 9$ & attendez je cherche ce dont je pourrais avoir besoin \\
\hline $20 \mathrm{M} 10$ & je n'ai encore rien à vous proposer... continuez s.v.p... \\
\hline $20 \mathrm{H} 10$ & attendez je vous dis \\
\hline
\end{tabular}




\begin{tabular}{|l|l|}
\hline $20 \mathrm{M} 11$ & ah oui, comment cela? \\
\hline $20 \mathrm{H11}$ & mais deux minutes \\
\hline
\end{tabular}

21 Nous sommes donc face à un genre, à savoir la conversation écrite entre homme et machine ; autre caractéristique celle-ci comporte des traits d'oralité.

\subsection{Les traits d'oralité}

22 L'écrit est, dans cet échange, le résultat de la production et mise en place de la parole de l'utilisateur. En d'autres termes, ces énoncés écrits sont le fruit de l'interaction quasi directe de l'utilisateur avec la machine. L'oral, quant à lui, livre un discours qui se construit, en incluant éventuellement ses propres corrections alors qu'ici ce type d'échange, via le clavier, fait perdre à l'oral son caractère fugace. Il faut noter qu'il n'y pas, ou peu, de distance entre l'élaboration de la chaîne écrite et son émission. Certaines différences structurales, d'ordre syntaxique ou lexical, qui opposent l'écrit à l'oral sont présentes dans le corpus. Il s'agit notamment des phénomènes suivants :

les interjections :

\begin{tabular}{|l|l|}
\hline 5M2 & PERMETTEZ-MOI QUE JE VOUS DEMANDE D'ÊTRE PLUS CLAIR... \\
\hline $5 \mathrm{H} 2$ & AH ??? \\
\hline
\end{tabular}

24 Le cas des points d'interrogation accompagnant cette interjection fera l'objet d'une analyse dans le chapitre 2.3 car la ponctuation est une caractéristique de ces interactions homme-machine (désormais IHM) que nous développerons .

\begin{tabular}{|l|l|}
\hline $9 M 8$ & $\begin{array}{l}\text { J'AI RELEVÉ } 8 \text { FICHES SUR «INTELLIGENCE ARTIFICIELLE », VOULEZ-VOUS TOUTES LES } \\
\text { CONSULTER? }\end{array}$ \\
\hline $9 H 8$ & BAH OUI \\
\hline $16 H 6$ & BAH PARLER AVEC TOI \\
\hline
\end{tabular}

les répétitions : il s'agit là, le plus souvent de répétitions particulières dans le sens où elles portent sur l'acceptation ou le refus de ce que propose la machine.

\begin{tabular}{|l|l|}
\hline $3 H 8$ & oui OUI JE VOUS SUIS \\
\hline $16 H 22$ & NON NON BYE \\
\hline $17 H 5$ & NON NON JE DÉCONNAIS \\
\hline
\end{tabular}


les énoncés formulant une indécision : bien qu'ils ne soient pas pleinement spécifiques de l'oral, doute, incertitude, hésitation, perplexité formulés dans certains énoncés sont les traces de la production de la parole et du sens; ; c'est une production qui se voit puisqu'elle est explicitée dans les propos mêmes de l'utilisateur. Ceci constitue donc un écrit exprimant un doute quant au résultat escompté.

\begin{tabular}{|l|l|}
\hline $3 M 1$ & ENTREZ VOTRE DEMANDE. \\
\hline 3 H1 & OUI MAIS COMMENT ? \\
\hline $3 H 4$ & JE SAIS PAS PEUT-ÊTRE AMBIGU \\
\hline 9M17 & VOUS N'AURIEZ PAS UN AUTRE CRITÈRE DE RECHERCHE QUE « PERTUBATIONS »? \\
\hline 9H17 & JE SAIS PAS LES MALADIES \\
\hline
\end{tabular}

- les niveaux de langue : beaucoup de termes issus de la conversation, du langage familier et des anglicismes ont été relevés :SALUT, BYE, WELL, OK, JE DÉCONNAIS, NON J'AI DÉJÀ DONNÉ, C'EST VRAIMENT TRÈS CHOUETTE COMME SYSTÈME, COOL,..., Les niveaux de langue et certains procédés énonciatifs sont davantage des faits de l'oral (quoiqu'il est vrai, certains écrivains recourent à ces procédés) comme le montrent ces extraits du corpus parmi les plus significatifs (c'est nous qui soulignons) :

\begin{tabular}{|l|l|}
\hline 4M5 & VOUS IMPORTE-T-IL DE CONSULTER CE QUE J'AI SUR « DISCOURS JURIDIQUE »? \\
\hline $4 H 5$ & OUI ON VERRA BIEN SI Y Y’A DE LA JURISPRUDENCE \\
\hline $4 H 10$ & C'EST DINGUE LE MANQUE DE COURTOISIE !!! \\
\hline 6 M10 & J'AI 28 FICHES SUR « PRATIQUES DE L'ÉCRIT », LES DÉSIREZ-VOUS TOUTES ? \\
\hline 6 H10 & C'EST COOL! \\
\hline $18 H 7$ & WELL FAUT PAS VOUS DÉTOURNER DU DROIT CHEMIN COMME ON DIT! \\
\hline
\end{tabular}

Les phénomènes d'enchaînements de mots sur un autre : c'est-à-dire quand l'utilisateur répond à un énoncé de la machine, sans le reprendre, mais en y réagissant ; ils sont très nombreux comme à l'oral :

\begin{tabular}{|l|l|}
\hline 9M11 & JE VOUS PRIE DE M'INDIQUER DE QUEL(LE) « LANGAGE »IL S'AGIT ? \\
\hline 9H11 & CELUI DES SOURDS MUETS \\
\hline $10 M 2$ & UN OUVRAGE SUR « HOMME-MACHINE »'EST CELA ? \\
\hline $10 H 2$ & NON SUR L'HYPERTEXTE \\
\hline
\end{tabular}




\begin{tabular}{|l|l|}
\hline 19M21 & J'ESPÈRE VOUS « REVOIR » SUR LING PROCHAINEMENT... \\
\hline \hline $19 H 21$ & DANS 6 MOIS ALORS \\
\hline
\end{tabular}

Ces phénomènes d'enchaînements d'un mot sur un autre, que nous sommes tentés de qualifier de dialogisme, sont les traces d'une conversation fonctionnant comme à l'oral.

les constructions syntaxiques : bien que ces dialogues soient écrits, ils font état d'une utilisation très particulière de constructions syntaxiques. Rupture, discontinuité ou enchaînement dans la construction d'une phrase, référence à un segment de la phrase de la machine, etc., ces éléments semblent bien montrer que la syntaxe utilisée dans les phrases des utilisateurs est différente de celle de l'écrit et s'apparente davantage à la conversation orale :

\begin{tabular}{|l|l|}
\hline $5 M 4$ & $\begin{array}{l}\text { JE REGRETTE MAIS SUR « DIT », JE NE SUIS PAS EN MESURE DE VOUS AIDER, PROPOSEZ-MOI } \\
\text { AUTRE CHOSE... }\end{array}$ \\
\hline $5 H 4$ & AUTRE CHOSE QUE DIT EN EFFET \\
\hline $10 M 9$ & DÉSIREZ-VOUS MENER UNE AUTRE RECHERCHE AVEC MOI? \\
\hline $10 H 9$ & OUI JE VOULAIS ENFIN SUR LA SOCIOLOGIE \\
\hline $15 M 2$ & UN OUVRAGE SUR « LANGUES GERMANIQUES » C'EST CELA ? \\
\hline $15 H 2$ & OUI C'EST CELA MAIS SURTOUT DES THÈSES \\
\hline $15 M 15$ & DONNEZ-MOI AU MOINS UN CONCEPT ? \\
\hline $15 H 15$ & C'EST QUOI UN CONCEPT \\
\hline $15 M 16$ & DONNEZ-MOI UN THÈME COMME «INTERACTION » OU « KINÉSIQUE », JE NE SAIS... \\
\hline $15 H 16$ & JE VOIS PAS FACILE \\
\hline
\end{tabular}

Et bien qu'il n'y ait pas de bégaiements d'actualisation, ni de lapsus, ces caractéristiques nous permettent d'inférer que nous sommes en présence d'une syntaxe propre au dialogue. F. DEBYSER (1989: 19) appelle ce phénomène une «créolisation graphique du français "; mais nous lui préférons le terme de réduction graphique de la langue en ceci qu'il exprime davantage l'idée selon laquelle ce type de support de la communication impose de faire court. Ces phénomènes produisent manifestement un effet de communication orale.

la chute du ne de la négation comme à l'oral est très fréquente :

\begin{tabular}{|l|l|}
\hline $3 H 9$ & JE SAIS PLUS MOI SUR DES CONDUITES AMBIGUËS \\
\hline $3 H 12$ & NON, J'AI RIEN EU SUR L'AMBIGUÏTÉ ! \\
\hline
\end{tabular}


Remarquons ici que la construction de la négation (avec absence du ne) dans les deux derniers exemples est typique de l'expression orale dont l'usage tend à se multiplier comme le montre aussi les niveaux de langue utilisés. La négation porte alors sur plus, rien , pas... ceci tend à se normaliser à l'oral alors que cet « oubli » constitue encore une grave faute dans un écrit soutenu.

Le métadiscours : c'est ici un discours qui porte sur l'échange (c'est nous qui soulignons):

\begin{tabular}{|l|l|}
\hline $12 M 1$ & BONJOUR JE SUIS UN SYSTÈME AUTOMATIQUE DE DIALOGUE, PERMETTEZ QUE JE VOUS AIDE... \\
\hline $12 H 1$ & UNE AUTOMATE QUI PARLE ON CROIT RÊVER ! \\
\hline $17 H 7$ & WELL FAUT PAS VOUS DÉTOURNER DU DROIT CHEMIN COMME ON DIT! \\
\hline $17 M 8$ & UN OUVRAGE SUR « DISCOURS JURIDIQUE » C'EST CELA ? \\
\hline $17 H 8$ & C'EST MOI QUI VOUS SUIT PLUS MAINTENANT \\
\hline $17 M 9$ & «MAINTENANT »??? \\
\hline $17 H 9$ & OU JAMAIS... \\
\hline $17 M 10$ & VOUS NE PARLIEZ PAS DE « DROIT » MAIS QUE CHERCHEZ-VOUS ALORS ? \\
\hline $17 H 10$ & $\begin{array}{l}\text { AH OUI BON DONNEZ MOI CE QUE VOUS AVEZ SUR LES MÉCANISMES DES GESTE ALLIÉS A CE } \\
\text { QU'ON VEUT EXPOSER }\end{array}$ \\
\hline $17 M 11$ & JE SUIS NAVRÉ MAIS JE N'AI AUCUNE FICHE CONCERNANT CETTE QUESTION. \\
\hline $17 H 11$ & RIEN SUR LES MÉCANISMES DES MOUVEMENTS COMMENT ÇA S'APPELLE? \\
\hline $17 M 12$ & VOUS VOULEZ PARLER DE « KINÉSIQUE »? \\
\hline $17 H 12$ & ET BIEN VOILA ON PEUT S'ENTENDRE \\
\hline
\end{tabular}

Les termes du métadiscours sont ici des références à un discours oral, des mots du discours de l'utilisateur qui font état du genre d'échange dont il affecte ou qualifie sa consultation (c'est nous qui soulignons) :

\begin{tabular}{|l|l|}
\hline 5M3 & FORMULEZ VOTRE DEMANDE DE FAÇON CLAIRE ET CONCISE S.V.P. ? \\
\hline 5H3 & MAIS JE N'AI ENCORE RIEN DIT \\
\hline 8M6 & VOULEZ-VOUS DIRE «LEXICOGRAPHIE»? \\
\hline
\end{tabular}




\begin{tabular}{|l|l|}
\hline $8 H 6$ & PUISQUE VOUS LE DITES \\
\hline $14 M 9$ & JE VOUS PRIE DE M'INDIQUER DE QUEL(LE) « ANALYSE »IL S'AGIT ? \\
\hline $14 H 9$ & JE VIENS DE VOUS DIRE SUR FLAUBERT \\
\hline $16 \mathrm{M} 6$ & QUE VOULEZ-VOUS EXACTEMENT ? \\
\hline $16 H 6$ & BAH PARLER AVEC TOI \\
\hline \hline $18 M 21$ & JE PEUX VOUS PROPOSER QUELQUE CHOSE SUR «PRATIQUES DE LECTURE »... \\
\hline $18 H 21$ & NON C'EST PAS CE QUE JE VOULAIS DIRE \\
\hline
\end{tabular}

37 Il faut noter que très souvent en français le terme dire est employé indifféremment pour : " signifier ", " écrire ", " exprimer ", " affirmer ", " exposer », " penser », etc., mais il semblerait que, dans ces énoncés, l'humain commente l'échange comme dialogique du fait des termes du discours qu'il utilise. Ainsi le premier exemple est significatif de ce fait car employer dans ce contexte : « dit » et non pas « écrit » ou « exprimé » ne signifie pas la même chose. L'utilisation qui est faite ici ne nous semble pas correspondre par exemple à l'emploi dans un écrit de l'expression : «Comme le dit X dans son article.. ».

\section{Analyse de l'utilisation de la ponctuation dans les énoncés de l'utilisateur}

Il faut remarquer l'absence très fréquente de toute ponctuation dans les énoncés de l'utilisateur :

\begin{tabular}{|l|l|}
\hline $3 H 6$ & VOUS N'AVEZ RIEN SUR DES DISCOURS AMBIGUS \\
\hline $3 M 7$ & LE CONCEPT DE « DISCOURS » RENVOIE À PLUSIEURS MOTS CLEFS, POURRIEZ-VOUS LE PRÉCISER \\
$?$
\end{tabular}

La ponctuation est un système particulier dans l'échange considéré. Aussi convient-il d'examiner de près cette question. Nous abordons ici le système ponctuationnel général ${ }^{3}$ et les pratiques du seul utilisateur. En effet, puisque les énoncés de M préexistent à l'interaction, ils ne nous intéressent ici que secondairement de ce point de vue. Notons seulement que la machine utilise une ponctuation dans presque tous ses énoncés. En revanche, la ponctuation dans les énoncés de l'humain est intéressante car elle met en œuvre un système particulier. Tout d'abord il faut indiquer que la ponctuation dans les énoncés de l'humain est très peu utilisée : 50 signes utilisés pour 334 " tours de parole » 
sur l'ensemble du corpus. De plus, elle est assez pauvre puisque les seuls signes de ponctuation utilisés par les utilisateurs sont :

- le point d'interrogation :

- utilisé seul (19),

- trois points d'interrogation successifs (7),

- le point d'exclamation :

- utilisé seul (12),

- trois points d'exclamation successifs (6),

les points de suspension (5)

- la virgule : une seule sur l'ensemble du corpus traité.

Il faut indiquer aussi qu'il n'y a aucun point, ni point virgule dans les énoncés des utilisateurs. Ceci peut s'expliquer dans ce type de conversation comme l'indique J. ANIS (1994: 90) dans son étude sur la ponctuation: "La rareté du point paraît refléter le mouvement discursif du dialogue, qu'il faut sans cesse prolonger et faire rebondir. Le point aurait une valeur conclusive trop forte. A l'inverse les points de suspension, avec différentes variantes, appellent à la poursuite de la conversation ». C'est ce que nous avons pu observer dans notre corpus. Ajoutons que l'absence du point virgule peut s'expliquer, quant à elle, par sa rareté dans l'usage contemporain de l'écrit. Ceci n'est donc pas étonnant dans cet écrit dont l'organisation est encore «floue ». Aucune règle n'en fixe l'usage !

41 Le tableau ci-dessous indique le nombre de signes de ponctuation utilisés par l'utilisateur dans chaque consultation.

\begin{tabular}{|c|c|c|c|c|c|c|c|c|c|c|c|c|c|c|c|c|c|c|c|c|c|}
\hline $\begin{array}{c}\mathbf{N}^{\circ} \text { de la } \\
\text { Consultation }\end{array}$ & 1 & 2 & 3 & 4 & 5 & 6 & 7 & 8 & 9 & 10 & 11 & 12 & 13 & 14 & 15 & 16 & 17 & 18 & 19 & 20 & 21 \\
\hline $\begin{array}{c}\text { Nombre } \\
\text { se } \\
\text { signes }\end{array}$ & 1 & 2 & 3 & 2 & 2 & 1 & 2 & 1 & 5 & 1 & 2 & 4 & 1 & 1 & 2 & 4 & 2 & 6 & 5 & 5 & $/$ \\
\hline
\end{tabular}

La moyenne de signes de ponctuation utilisés est donc de deux signes par consultation. De plus, si l'on excepte les consultations $n^{\circ} 1,8$ et 21 qui ne possèdent aucune marque de ponctuation dans les énoncés de l'utilisateur, cette moyenne est alors de 2,7 signes par consultation et moins de 0,2 signes par tour de "parole». Bien que ces chiffres ne signifient pas grand chose, ils attestent tout de même d'un fait important : la très faible utilisation de la ponctuation dans les énoncés des usagers. Il faut noter que ces signes sont très souvent les marques de l'affect que manifestent le plus souvent les énoncés de l'utilisateur. Ils ont davantage une valeur sémantique forte qu'une véritable valeur ponctuationnelle :

\begin{tabular}{|l|l|}
\hline $20 M 17$ & UNE AUTRE RECHERCHE PEUT-ÊTRE ? \\
\hline \hline $20 H 17$ & C'EST PAS SUR LE LEXIQUE CES FICHES !!!... \\
\hline
\end{tabular}

43 Le triplement des points d'interrogation et d'exclamation ( $c f$. respectivement les exemples $5 \mathrm{H} 2$ et $4 \mathrm{H} 10$ ) a une valeur sémantique forte qui exprime des sentiments ou de vives réactions dans ces écrits.

La ponctuation possède un statut auxiliaire dans ces dialogues. Elle produit une information redondante ou complémentaire comme J. ANIS a pu le montrer à partir d'autres corpus (1994 : 95). Dans ce type de dialogue, la ponctuation possède une valeur singulière qui diffère de celle des écrits traditionnels. Nous avons relevé trois types de valeur qui en fait se résument à deux valeurs comme nous allons le voir : 
démarcative :

\begin{tabular}{|l|l|}
\hline 10M1 & BONSOIR, VEUILLEZ ÉNONCER VOTRE REQUÊTE. \\
\hline \hline $10 H 1$ & SLT, JE VOUDRAIS DES TRAVAUX SUR L'HYPERTEXTE \\
\hline
\end{tabular}

La virgule a ici une valeur démarcative et sépare les deux actes de langage. Cependant nous constatons qu'il existe une véritable économie des signes à valeur démarcative (pour ne pas dire une absence) comme on peut en user habituellement à l'écrit. C'est la seule virgule utilisée par un usager sur l'ensemble du corpus! Rappelons que nous n'avons relevé aucun point, ni point virgule dans tout le corpus, soit sur plus de 300 tours de parole! Cette valeur démarcative ne nous semble donc pas être pertinente pour la description de notre corpus. En fait, et l'utilisation de cette seule virgule ne peut le contredire, l'analyse du corpus montre qu'il n'existe pas à proprement parler de ponctuation à valeur démarcative dans ces énoncés.

En revanche le corpus fait état d'une ponctuation à valeur :

« sémantique «: cette dernière indique très formellement le statut de la phrase :

\begin{tabular}{|l|l|}
\hline $9 m 16$ & PRÉCISEZ-MOI DONC CE QUE VOUS VOULEZ QUE JE CIBLE DAVANTAGE VOTRE REQUÊTE... \\
\hline $9 H 16$ & Le langage et ses perturbations? \\
\hline $12 \mathrm{M} 11$ & notre collaboration a été fructueuse, j'ai une fiche sur « bilinguisme \\
\hline $12 \mathrm{H} 11$ & fructueuse? \\
\hline $15 \mathrm{M} 14$ & vous avez écrit « dire » êtes-vous sûr? \\
\hline $15 \mathrm{H} 14$ & n'importe quoi! \\
\hline
\end{tabular}

\section{expressive :}

\begin{tabular}{|l|l|}
\hline $4 M 10$ & DONNEZ-MOI AU MOINS UN CONCEPT... \\
\hline $4 H 10$ & C'EST DINGUE LE MANQUE DE COURTOISIE !!! \\
\hline \hline $5 M 2$ & PERMETTEZ-MOI QUE JE VOUS DEMANDE D'ÊTRE PLUS CLAIR... \\
\hline $5 H 2$ & AH ??? \\
\hline \hline $14 M 7$ & ESSAYEZ D'ÊTRE PLUS EXPLICITE JE VOUS PRIE... \\
\hline $14 H 7$ & PLUS EXPLICITE ??? \\
\hline $12 M 3$ & JE NE VOUS SUIS PAS VRAIMENT... QUE VOULEZ-VOUS ? \\
\hline
\end{tabular}




\begin{tabular}{|l|l|}
\hline $12 H 3$ & AH LA SCIENCE NE S'ARRÊTERA JAMAIS !!! \\
\hline \hline $18 M 17$ & DÉSIREZ-VOUS MENER UNE AUTRE RECHERCHE AVEC MOI ? \\
\hline $18 H 17$ & OUI MAIS FAUT QU'ÇA MARCHE MIEUX QU'ÇA !!! \\
\hline
\end{tabular}

50 Nous insistons sur le fait que l'utilisation de ces signes, à savoir le triplement des points d'interrogation et d'exclamation marquent une vive réaction, la surprise ou l'étonnement et l'incompréhension de l'utilisateur. La ponctuation à valeur expressive est très utilisée dans notre corpus. Elle est employée par l'utilisateur (et par la machine) pour pallier certains manques par rapport à la production orale tels que : froncement de sourcils, intonation, gestuel,..., toutes les manifestations qui peuvent accompagner la parole pour signifier ou renforcer ce qui est dit.

\section{Conclusion}

51 Ces analyses nous ont conduit à tirer certaines conclusions sur la nature des énoncés produits qui mériteraient d'être confirmer, infirmer ou simplement affiner à partir de l'analyse d'autres corpus. Elles ne peuvent en aucun être étendues à l'ensemble des situations dans lesquelles des individus "dialoguent» avec une machine. En effet, nos recherches en ce domaine nous ont appris qu'il n'existait pas une bonne manière d'aborder la question de l'IHM. Cependant, en nous en tenant à cette expérimentation, nous pouvons affirmer que cet échange est une forme nouvelle intégrant certaines caractéristiques distinctives de l'écrit et de l'oral. Certains énoncés utilisent même ces deux formes au sein du même énoncé. Aussi nous pensons comme l'affirme F. DEBYSER (1989: 18) que: "Quoi qu'il en soit, la convergence de tous ces facteurs aboutit à l'affichage d'énoncés bâtards caractérisés massivement par le recours à des niveaux de langue familiers ou à des procédés linguistiques typiques de la langue orale ». Cette interaction comporte donc de nombreuses marques propres de l'oral (les répétitions, les interjections, les niveaux de langue familiers, chute du ne, etc.) qui produisent un effet de dialogue. Ces phénomènes sont autant d'éléments qui rythment la production de la parole de l'utilisateur. Ils constituent les marques graphiques des transcriptions écrites constituant le corpus. Les caractéristiques de cet échange écrit sont elles aussi assez nombreuses. Il s'agit notamment de l'utilisation d'abréviations, de la réduction de certains mots et de l'emploi «marginal » de la ponctuation qui acquiert alors une valeur expressive ou sémantique. Ces faits fondent une mise en scène graphotextuelle. Pour ce qui est imposé par l'interface, il faut noter principalement le temps de transmission très court, la mécanisation des tours de parole et l'absence de pause.

Cet échange écrit, comme l'analyse du corpus le révèle, s'apparente davantage à un échange oral qu'à la communication écrite, mais incontestablement il constitue une nouvelle forme d'interaction. Ainsi, il emprunte à l'écrit et à l'oral, des marques qui leur sont spécifiques et qui caractérisent l'un et l'autre mode, pour créer un usage nouveau. Le terme de dialogue homme-machine dans le cadre de cet échange semble donc adapté du fait que l'utilisateur emploie des registres de langue comme dans une conversation. De plus, la syntaxe de l'oral, qui ne peut se confondre avec celle de l'écrit, est ici remarquable au sens strict. Dans ce sens, D. LUZZATI (1989) parle, d'oral-écrit, et 
pourtant cette interaction demeure strictement un échange écrit possédant des marques d'oralité, n'impliquant que deux participants, l'utilisateur et la machine. La machine crée une nouvelle forme de dialogue que nous avons examinée dans son rapport sociocontextuel, en montrant comment la langue qui n'est pas un système stable et figé évolue par et au travers des utilisateurs vis-à-vis de ces nouveaux supports de communication. La langue tient bien sûr une place essentielle dans la recherche sur le DHM mais nous pensons qu'il faut nécessairement prendre en compte les phénomènes pragmatiques c'est-à-dire qui relèvent de l'étude de l'utilisateur en situation de communication, en situation même de dialogue, de l'usage qu'il fait de la langue.

\section{BIBLIOGRAPHIE}

ANIS, J. 1993, « L'écriture à sa place », dans Linx n² 28, Paris X, Nanterre, Paris, 53-57.

ANIS, J. 1994, «Pour une graphématique des usages : le cas de la ponctuation dans le dialogue télématique », dans Linx n 31, Paris X, Nanterre, Paris, 81-97.

ANIS, J. 1998, Texte et ordinateur : l'écriture réinventée ? De Boeck, Bruxelles.

CATACH, N. 1994, La ponctuation, Presses Universitaires de France, « Que sais-je ? », Paris, 96 pages.

DEBySER, F. 1989, « Télématique et enseignement du français », dans LANGUE FRANÇAISE nº 83, Larousse, Paris, 14-31.

DOPPAGNE, A. 1984, La bonne ponctuation : clarté, précision, efficacité de vos phrases, Duculot, Paris, 192 pages.

FAURE, C. et alii, 1994, Advances in Handwriting and Drawing : a Multidisciplinary Approach, Europia, Paris, 217 pages.

GRICE, H. P. 1975, Logic and Conversation, in COLE P., MORGAN J. L. (Eds) 1975.

LUZZATI, D. 1989, Recherches sur le dialogue homme-machine : modèles linguistiques et traitements automatiques, Thèse d'État, Paris III.

LuZZATI, D. 1991, « Oralité et intéractivité dans un écrit Minitel » dans LANGUE FRANÇAISE n 89 «L'oral dans l'écrit », Larousse, Paris, 124 pages.

LuZZATI, D. et alii, 1997, Le dialogique, colloque international sur les formes philosophiques, linguistiques, littéraires et cognitives du dialogue, Université du Maine, 15-16 septembre 1994, PETER LANG, PARIS, 402 PAGES.

Morel, M.-A. 1988, (Resp.), Analyse linguistique de dialogues homme/machine (Tome 1), Publications de la Sorbonne Nouvelle, Paris, Tome $1: 292$ pages, Tome 2:371 pages.

SABAH, G. et alii, 1997, Machine, Langage et Dialogue, Collection Figures de l'interaction, Paris, L'Harmattan, 342 pages.

VION, R. 1992, La communication verbale, (Analyse des interactions), Hachette, Paris, 302 pages. 
WABLE, T. 1998, Processus interactifs dans le dialogue homme-machine. Analyse des images identitaires, de la tâche et des dysfonctionnements lors d'une interrogation de base de données bibliographiques, Thèse de Doctorat NR, UPRESA CNRS 6065 DYALANG, Université de Rouen, Mont-Saint-Aignan, 512 pages.

Zock, M. 1991, « La génération interactive de langage : comment visualiser le passage de l'idée à la phrase ", Texte et ordinateur : les mutations du lire écrire, numéro spécial de LINX, Centre de Recherches Linguistiques de Paris X Nanterre, 249-267.

\section{NOTES}

1. Nous avons abordé dans notre thèse (1998) les spécificités de cette communication.

2. Abréviations utilisées $: H=$ Homme et $M=$ Machine.

3. En matière de ponctuation, du lexique des règles typographiques en usage à l'Imprimerie nationale aux règles préconisées par le bon usage, les ouvrages sont nombreux. Nous nous référons ici à l'ouvrage de N. Catach (1994) qui analyse la fonction de la ponctuation.

\section{RÉSUMÉS}

Cet article repose sur l'analyse d'un corpus de dialogues, recueilli au cours d'une simulation expérimentale, entre un utilisateur et une machine lors de l'interrogation d'une base de données bibliographiques. L'humain interroge une machine qui doit l'aider à retrouver l'information qu'il désire. Il s'agit ici de définir les spécificités de cet échange notamment en analysant les formes de l'écrit et de l'oral qu'il tend à prendre. Est-ce un dialogue ou une communication écrite ? Quelle est la structure de cet échange? Quels sont ses constituants et ses caractéristiques?

This article relies on the analysis of experimental simulated dialogues involving a user and a machine during a bibliographic data base inquiry. The human being asks an interface designed to help him to get the desired information. The point here is to define the specificities of this particular dialogue by analysing the written and oral forms it can take. Is it a dialogue or a written communication? What is the structure of this exchange? What are its elements and characterisrics?

\section{INDEX}

Mots-clés : dialogue homme-machine, dialogisme, interaction, oral/écrit, ponctuation à valeur sémantique, ponctuation à valeur expressive, système ponctuationnel, utilisateur

Keywords : man/machine dialogue, dialogism, written/oral forms, semantic punctuation role, expressive punctuation role, ponctuation logic, user 


\section{AUTEUR}

\section{THIERRY WABL}

UPRESA CNRS 6065 DYALANG

Université de Rouen, IRED,

Institut de recherches et de documentation en sciences sociales

7, rue Thomas Becket

76821 Mont-Saint-Aignan cedex

thierry.wable@normandnet.fr 